\title{
Impact of heart rate on diagnostic accuracy of second generation 320-detector computed tomography coronary angiography
}

\author{
Nitesh Nerlekar ${ }^{1}$, Brian S. Ko ${ }^{1}$, Arthur Nasis ${ }^{1}$, James D. Cameron ${ }^{1}$, Michael Leung ${ }^{1}$, Adam J. Brown ${ }^{1}$, \\ Dennis T. L. Wong', Philip J. Ngu ${ }^{1}$, John M. Troupis ${ }^{1,2}$, Sujith K. Seneviratne ${ }^{1}$ \\ ${ }^{1}$ Monash Cardiovascular Research Centre, Department of Medicine (Monash Medical Centre) Monash University and Monash Heart, Monash \\ Health, 246 Clayton Road, Clayton, 3168 VIC, Australia; ${ }^{2}$ Department of Diagnostic Imaging, MMC, Southern Health, Melbourne, Australia \\ Contributions: (I) Conception and design: N Nerlekar, A Nasis, BS Ko; (II) Administrative support: JD Cameron, SK Seneviratne; (III) Provision of \\ study material or patients: None; (IV) Collection and assembly of data: N Nerlekar, AJ Brown, PJ Ngu; (V) Data Analysis and interpretations: N \\ Nerlekar, M Leung, DT Wong, JM Troupis, SK Seneviratne; (VI) Manuscript writing: All authors; (VII) Final approval of manuscript: All authors. \\ Correspondence to: Dr Nitesh Nerlekar. MonashHeart, MonashHealth, 246 Clayton Road, Clayton, VIC 3168, Australia. \\ Email: nitesh.nerlekar@monashhealth.org.
}

Objective: To assess the impact of elevated heart rate (HR) on the diagnostic accuracy and image quality of second-generation 320-detector computed tomography coronary angiography (320-CTCA).

Methods: Consecutive patients with suspected coronary disease referred for invasive coronary angiography (ICA) were prospectively recruited and underwent 320-CTCA. Pre-scan beta-blockers were administered if native $\mathrm{HR}>80 \mathrm{bpm}$ and post-scan cohorts stratified by traditional (HR $\leq 60 \mathrm{bpm}$ ) and elevated HR (61-80 bpm). A wider phase window was used for the elevated HR group (30-80\%). 320-CTCA and ICA were analyzed by independent readers blinded to other data. Significant disease was defined as $\geq 50 \%$ visual stenosis on ICA. Uninterpretable segments by 320-CTCA were considered to be significant on an intentionto-diagnose principle. Image quality was assessed by 5 -point Likert score.

Results: Of 107 patients studied (1,662 segments), there was no significant difference in sensitivity, specificity, positive and negative predictive value between patients with $\mathrm{HR} \leq 60 \mathrm{bpm}(\mathrm{n}=55)$ vs. HR 61-80 bpm ( $\mathrm{n}=52)$ : 97\%, 88\%, 95\%, 94\% vs. 100\%, 88\%, 95\%, 100\%; Receiver operator characteristic-area under the curve 0.93 vs. $0.94, \mathrm{P}=0.82$ ). Overall per-patient diagnostic accuracy was $96 \%$ in both groups with no significant difference in interpretable segments (Likert $\geq 2)$ or median radiation dose $(2.4 \mathrm{mSv} v s .2 .7 \mathrm{mSv}$, $\mathrm{P}=0.35)$. Only 4/1,662 (0.2\%) segments were uninterpretable by motion artefact in the whole cohort.

Conclusions: In patients with $\mathrm{HR}>60$ and up to $80 \mathrm{bpm}$, second generation 320-CTCA provides comparably adequate diagnostic accuracy to $\mathrm{HR} \leq 60$ without significantly impacting upon overall segmental evaluability.

Keywords: Multi-detector CT; 320 row CT coronary angiography; diagnostic accuracy; sensitivity; specificity; positive predictive value; negative predictive value; image quality

Submitted Dec 05, 2016. Accepted for publication Jan 20, 2017.

doi: $10.21037 / \mathrm{cdt} .2017 .03 .05$

View this article at: http://dx.doi.org/10.21037/cdt.2017.03.05

\section{Introduction}

The high diagnostic accuracy of 320 detector-row computed tomography coronary angiography (320-CTCA) in patients with suspected coronary artery disease (CAD) has been demonstrated in multiple single-centre trials, when compared with invasive coronary angiography (ICA) (1-4). In contrast with traditional 64-detector CT, 320-CTCA enables complete acquisition of the coronary tree in a single gantry rotation. However, 320-CTCA using first generation scanners requires strict heart rate $(\mathrm{HR})$ control $(\leq 60$ beats per minute) due to a limited effective temporal resolution 
of $175 \mathrm{msec}$, which is similar to traditional 64 -detector systems (5). To achieve strict HR control, negative chronotropes (most commonly beta-blockers) are often administered via oral or intravenous route. This may increase patient length of stay, time to 320-CTCA, and may be contraindicated in a proportion of patients (6-10).

Imaging at higher HR (11-14) is often associated with motion artifacts resulting in suboptimal image quality (15). It is also associated with increased radiation exposure due to requirement for multiple gantry rotations (13), or wider padding around a mid-diastolic phase.

Second generation 320-CTCA has an effective temporal resolution of $137.5 \mathrm{msec}$ with half-segment reconstruction. This has the potential to image the entire coronary tree at elevated HR with a single gantry rotation at $0.5 \mathrm{~mm}$ slice collimation with complete $16 \mathrm{~cm}$ cranio-caudal coverage. Recent studies using second-generation 320CTCA have demonstrated superior image quality at varying HR (11) and lower radiation dose (12) when compared with first-generation scanners (14). Data on the diagnostic performance in comparison to ICA at low and high HR is lacking. This study's primary aim is to evaluate the diagnostic accuracy of second-generation 320-CTCA compared to ICA in patients with traditionally controlled $(\mathrm{HR} \leq 60 \mathrm{bpm})$ and elevated HR (61-80 bpm). The secondary aim is to determine the impact of HR control on image quality and radiation exposure.

\section{Methods}

\section{Study population}

Consecutive patients referred for clinically mandated ICA to a tertiary referral centre (Monash Heart, Monash Medical Centre, Melbourne, Australia) between November 2012 and March 2014 for evaluation of suspected CAD were prospectively screened. Patients were excluded if they had known obstructive CAD as determined on invasive angiography ( $\geq 50 \%$ stenosis), coronary artery bypass grafting, previous coronary stenting, advanced atrioventricular block, atrial or ventricular arrhythmia, decompensated heart failure, steroid-dependent asthma or renal insufficiency (eGFR $<60 \mathrm{~mL} / \mathrm{min} / 1.73 \mathrm{~m}^{2}$ ).

All patients gave written consent prior to inclusion in the study. After informed consent, all eligible patients who did not fulfill exclusion criteria underwent research 320-CTCA within one week of ICA. No patients required intervention between ICA and 320-CTCA. The institutional review board approved the study and all recruited patients provided signed informed consent.

\section{ICA protocol and interpretation}

ICA was performed via standard techniques by either radial or femoral approach at operator discretion. ICA images were visually evaluated by an interventional cardiologist blinded to clinical data and CTCA results by visual stenosis grade. Coronary segments were classified according to the modified American Heart Association 17-segment model (16) and significant stenosis was defined as $\geq 50 \%$ reduction of maximal coronary luminal diameter.

\section{0-CTCA protocol and interpretation}

Upon arrival in the CTCA department, HR was evaluated. Patients with baseline HR $\leq 80 \mathrm{bpm}$ underwent 320 CTCA acquisition at their native HR; for patients with HR $>80 \mathrm{bpm}, 25 \mathrm{mg}$ oral metoprolol or $5 \mathrm{mg}$ oral ivabradine was administered with repeat dosing at 30-minute intervals to allow imaging to be performed at $\mathrm{HR} \leq 80 \mathrm{bpm}$. Ontable intravenous metoprolol was administered if the HR increased above $>80 \mathrm{bpm}$. All studies were performed on a second-generation 320-detector row system (AquilionOne VISION, Toshiba Medical Systems, Tokyo, Japan). Nitroglycerin $400 \mu \mathrm{g}$ sublingually was administered 1 minute before contrast injection. A bolus of $75 \mathrm{~mL}$ of $100 \%$ Iohexal (Omnipaque 350) was administered at $6 \mathrm{~mL} / \mathrm{s}$ followed by a $50 \mathrm{~mL}$ normal saline chaser. Scanning was manually triggered when peak contrast enhancement in the left ventricle was observed with no enhancement in the right ventricle. Scans were performed via an axial technique with detector collimation of $320 \mathrm{~mm} \times 0.5 \mathrm{~mm}$ and no requirement for table movement due to $16 \mathrm{~cm}$ cranio-caudal coverage. Tube current was determined with the use of automatic exposure control (SUREExposure3D, Toshiba medical systems) on the basis of X-ray attenuation on anterior-posterior and lateral scout images and the reconstruction kernel. Tube potential was manually set by the radiographer with default at $100 \mathrm{kVp}$ and adjusted to 120 or $135 \mathrm{kVp}$ when the automatic tube current selected was maximum. Gantry rotation time was $275 \mathrm{msec}$ with effective temporal resolution of $137.5 \mathrm{msec}$. Scans were performed with prospective electrocardiographic triggering using $70-85 \%$ of the phase window at $\mathrm{HR}<70 \mathrm{bpm}$ and $30-80 \%$ when HR $>70 \mathrm{bpm}$. Image acquisition was restricted to single beat acquisition (multi-beat acquisition 
was not performed). Images were reconstructed with a 512 $\times 512$ matrix, $0.5 \mathrm{~mm}$ thick sections and $0.25 \mathrm{~mm}$ increments using kernel $\mathrm{FC} 43$, iterative reconstruction with adaptive iterative dose reduction 3D (AIDR3D, Toshiba Medical Systems) standard and asymmetric cone beam reconstruction (17). Mean effective radiation exposure was derived from the doselength product multiplied by a conversion co-efficient for the chest $(\mathrm{DLP} \times 0.014 \mathrm{mSv} / \mathrm{mGy})(18)$.

CTCA images were visually assessed by consensus by two CT trained cardiologists. Readers were blinded to clinical and ICA datasets. Reconstructed images were reviewed on a computer-based platform (Vitrea FX 2.0, Vital Images, Minnetonka, Minnesota) with the best phase chosen for interpretation. Quantification was performed according to standardized 17-segment model (16) for all segments $\geq 1.5 \mathrm{~mm}$ diameter. Significant stenosis was defined as $\geq 50 \%$ reduction of the maximal luminal diameter. Image quality was assessed at per-segment level according to a previously published 5-point Likert score (19): 1=poor, impaired image quality limited by excessive noise or poor vessel wall definition; 2 =adequate, reduced image quality with poor vessel wall definition or excessive image noise, limitations in low contrast resolution remain evident; 3 =good, impact of image noise, limitations of low contrast resolution and vessel margin definition are minimal; 4=very good, good attenuation of vessel lumen and delineation of vessel walls, relative image noise is minimal, coronary wall definition and low contrast resolution well maintained and $5=$ excellent, excellent attenuation of the vessel lumen and clear delineation of the vessel walls, limited perceived image noise. If the segment was described as poor, the predominant artifact was identified as calcification, motion or noise. Non-interpretable segments were regarded as containing severe stenosis.

\section{Statistical analysis}

Continuous variables are reported as mean \pm standard deviation or median with interquartile range. Normality of variables was assessed visually by plotting histograms and statistically by the Shapiro-Wilk test. Variables were compared using a Student t-test, Mann-Whitney or Kruskal Wallis as appropriate. Categorical variables were compared with a chi-square or Fishers exact test. Interobserver agreement between CT readers was measured the Cohen $\kappa$ test with the scale: $\kappa<0.2$ indicates poor agreement, $0.21-$ 0.40 fair agreement, $0.41-0.60$ moderate agreement, $0.61-$ 0.80 good agreement, 0.81-1.00 excellent agreement (12).
Diagnostic accuracy of 320-CTCA compared to ICA are presented as sensitivity, specificity, positive predictive value (PPV) and negative predictive values (NPV); with precision expressed as $95 \%$ confidence intervals as well as by receiver operator characteristic (ROC) analysis comparison and chi-square test (20). Computation of confidence limits for vessels and segments were calculated by generalized estimating equations to account for within-patient clustering (21). Assessment of the Likert score of image quality probability according to HR group was evaluated by ordered logistic regression. Binary logistic regression was performed to assess the image quality difference between high and low HR groups against interpretable (Likert >2) vs. non-interpretable segments. Study analysis was performed on an intention-to-diagnose principle (3) to represent 'realworld' practices. A P value $<0.05$ was considered statistically significant. Statistical analysis was performed using Stata 14/MP (StataCorp, College Station, Texas, USA).

\section{Results}

\section{Patient population}

Of 885 consecutive patients undergoing ICA, 107 met inclusion criteria for study participation (Figure 1). During scan acquisition, 55 patients (Group 1) met traditional rate control criteria with HR $\leq 60 \mathrm{bpm}$ and 52 (Group 2) had elevated HR from 61-80 bpm. The mean HR in Group 1 was $52 \pm 5$ bpm compared to $69 \pm 8$ bpm in Group $2(\mathrm{P}<0.001)$. The range of heart rate in the study was from minimum $37 \mathrm{bpm}$ to maximum $80 \mathrm{bpm}$. Twenty-nine patients required rate control medication due to baseline $\mathrm{HR}>80 \mathrm{bpm}$ with 17 patients in Group 1 (31\%) and 12 in Group 2 (21\%), $\mathrm{P}=0.25$. Baseline demographics and 320-CTCA scan parameters are shown in Tables 1 and 2 respectively.

\section{Invasive angiography}

There were 215 significant stenoses identified with 110 in Group 1 and 105 in Group 2 ( $\mathrm{P}=0.93)$. Single vessel disease was present in 36 patients, double vessel disease in 28 patients and triple vessel disease in 10 patients in the entire cohort. There were no complications related to ICA.

\section{Diagnostic accuracy}

\section{Disease prevalence}

There was a high prevalence of significant CAD $(\geq 50 \%$ 


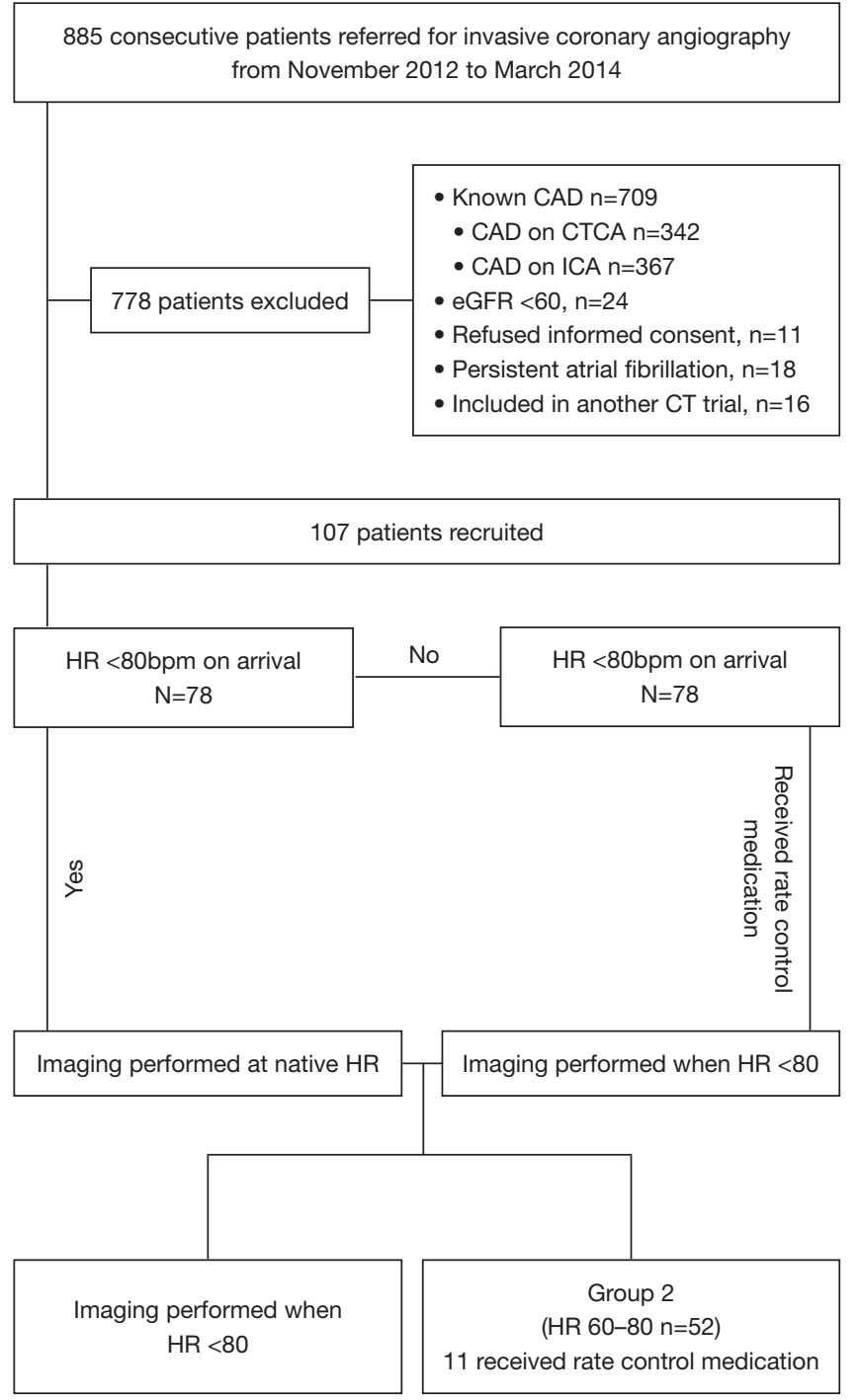

Figure 1 Group selection. CTCA, computed tomography coronary angiography; CAD, coronary artery disease; ICA, invasive coronary angiography; eGFR, estimated glomerular filtration rate; $\mathrm{HR}$, heart rate.

stenosis in any coronary segment) with per-patient prevalence in Group 1 in 39/55 (70.9\%) on ICA vs. 37/55 $(67.2 \%)$ on $320-\mathrm{CTCA}(\mathrm{P}=0.68)$. In Group 2 the prevalence of significant disease was $35 / 52$ patients $(67.3 \%)$ on ICA $v$ s. $34 / 52(65.3 \%)$ on $320-\mathrm{CTCA}(\mathrm{P}=0.84)$.

\section{Per-segment analysis}

In Group 1,855 segments were evaluated by 320-CTCA and 807 segments in Group 2. At a per-segment level in Group 1 vs. Group 2, there was no significant difference in
Table 1 Baseline clinical demographics

\begin{tabular}{lccc}
\hline Characteristic & $\begin{array}{c}\text { Group 1 (HR } \\
\leq 60)(\mathrm{n}=55)\end{array}$ & $\begin{array}{c}\text { Group 2 (HR } \\
60-80)(\mathrm{n}=52)\end{array}$ & P value \\
\hline Age $(\mathrm{y})$ & $63 \pm 10$ & $63 \pm 9$ & 0.99 \\
$\mathrm{BMI}(\mathrm{m} / \mathrm{kg})^{2}$ & $28.5 \pm 4.3$ & $28.3 \pm 5$ & 0.81 \\
Males & $36(65 \%)$ & $32(62 \%)$ & 0.35 \\
Hypertension & $34(62 \%)$ & $30(55 \%)$ & 0.47 \\
Hyperlipidaemia & $24(44 \%)$ & $25(48 \%)$ & 0.76 \\
Diabetes & $10(18 \%)$ & $12(23 \%)$ & 0.59 \\
Smoker & $24(44 \%)$ & $23(44 \%)$ & 0.93 \\
Family history of IHD & $26(47 \%)$ & $24(44 \%)$ & 0.78 \\
Baseline beta blocker & $23(42 \%)$ & $19(37 \%)$ & 0.58 \\
\hline $\begin{array}{l}\text { Values are mean } \pm \text { SD or } \mathrm{n}(\%) . \text { BMI, body mass index; IHD, } \\
\text { ischaemic heart disease }\end{array}$ &
\end{tabular}

Table 2 320-CTCA scan parameters

\begin{tabular}{lccc}
\hline Parameter & $\begin{array}{c}\text { Group 1 (HR } \\
\leq 60)(\mathrm{n}=55)\end{array}$ & $\begin{array}{c}\text { Group 2 (HR } \\
60-80)(\mathrm{n}=52)\end{array}$ & P value \\
\hline $\begin{array}{l}\text { Acquisition heart rate } \\
\text { (bpm), (mean } \pm \mathrm{SD})\end{array}$ & $52 \pm 5$ & $69 \pm 8$ & $<0.001$ \\
$\begin{array}{l}\text { Received rate control } \\
\text { medication, } \mathrm{n}(\%)\end{array}$ & $17(31 \%)$ & $12(23 \%)$ & 0.36 \\
$\begin{array}{l}\text { Tube current }(\mathrm{mA}), \\
\text { (mean } \pm \text { SD) }\end{array}$ & $590 \pm 191$ & $568 \pm 178$ & 0.57 \\
$\begin{array}{l}\text { Tube Voltage, kVp, } \\
\text { median (IQR) }\end{array}$ & $100(100-120)$ & $100(100-120)$ & 0.51 \\
$\begin{array}{l}\text { Radiation dose (mSv), } \\
\text { median (IQR) }\end{array}$ & 2.45 & 2.70 & 0.35 \\
\hline
\end{tabular}

the sensitivity ( $89 \%$ vs. $84 \%, \mathrm{P}=0.78$ ), specificity ( $95 \%$ vs. $96 \%, \mathrm{P}=0.54)$, PPV (73\% vs. $76 \%, \mathrm{P}=0.57)$ and NPV (98\% vs. $97 \%, \mathrm{P}=0.28$ ) (Table 3). Overall per-segment accuracy was $94 \%$ in both groups.

\section{Per-vessel analysis}

There were 220 vessels analysed in Group 1 and 208 in Group 2. Overall accuracy was $92 \%$ in the Group 1 and $95 \%$ in Group 2. There was no significant difference in sensitivity (94\% vs. 98\% $\mathrm{P}=0.92)$, specificity ( $91 \%$ vs. $94 \%$, $\mathrm{P}=0.35)$, PPV ( $81 \%$ vs. $86 \% \mathrm{p}=0.32$ ) or NPV ( $97 \%$ vs. $99 \%$ $\mathrm{P}=0.37)$ between groups. 
Table 3 Diagnostic accuracy parameters of 320-CTCA

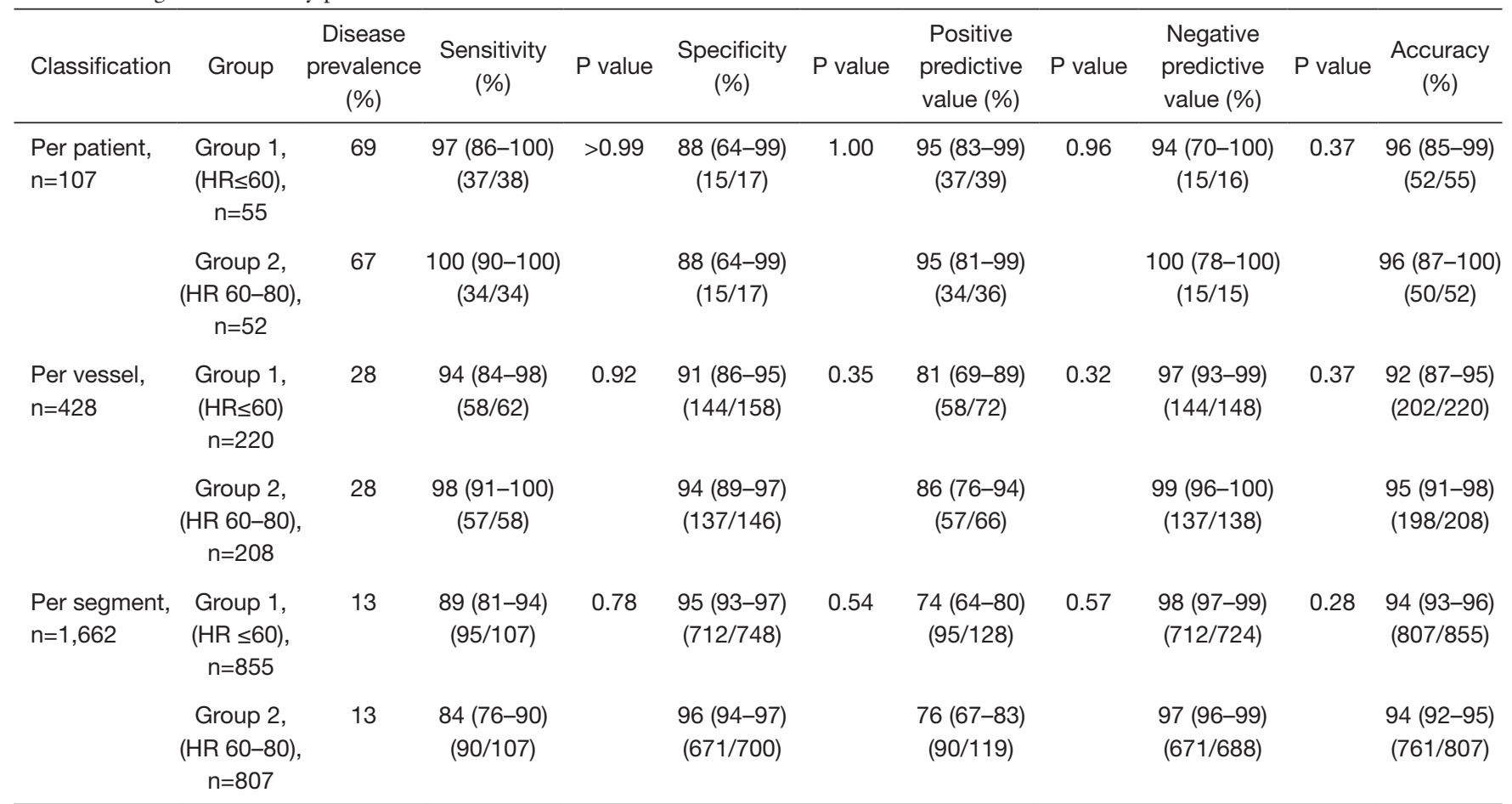

Diagnostic parameter values represented by parameter percentage and respective $95 \%$ confidence intervals with raw numbers for parameter calculation below.

\section{Per-patient analysis}

The overall per-patient diagnostic accuracy across all 107 patients was $96 \%$. There was no significant difference in accuracy parameters in the Group 1 vs. Group 2. Sensitivity was $97 \%$ vs. $100 \%(\mathrm{P}>0.99)$, specificity $88 \%$ vs. $88 \%$ $(\mathrm{P}>0.99)$, $\mathrm{PPV} 95 \%$ vs. $95 \%(\mathrm{P}=0.96)$ and $\mathrm{NPV} 94 \%$ vs. $100 \%(\mathrm{P}=0.37)$.

ROC analysis at a per-patient level found no difference in diagnostic accuracy between groups (Group $10.93 \mathrm{vs}$. Group 2 0.94, $\mathrm{P}=0.82$ ).

\section{Image quality}

The interobserver agreement between readers was excellent $(\kappa=0.84)$. Of 1,662 segments evaluated on 320-CTCA, $1,632(98 \%)$ were interpretable (defined as Likert score $\geq 2$ ). There was a significant difference in image quality between groups, with median Likert score 4 (IQR 3-4) in Group 1 vs. 4 (IQR 3-4) in Group $2(\mathrm{P}<0.01)$ with a larger proportion of 'adequate' (Likert score $=2$ ) segments in the Group 2 (73 vs. 21 in Group 1, $\mathrm{P}<0.01$ ) (Table 4).

Ordered logistic regression demonstrated a decrease in image quality, with increasing Likert score for each unit increase in HR ( $\beta$ coefficient -0.371 (95\% CI: -0.56 , $-0.17, \mathrm{P}<0.001)$ with reducing probabilities across all levels of Likert score. However when assessed according to interpretable $v s$. non-interpretable segments, there was no significant difference in the log odds of higher image quality in Group 1 vs. Group 2 ( $\beta$ coefficient -0.19 (95\% CI: -1.00, $0.61, \mathrm{P}=0.64$ ) (Table 4).

There was no difference in the number of poor or noninterpretable segments between groups $(\mathrm{P}=0.13)$ with assessment of the best CT phase. Of these 30 poor/noninterpretable segments in the entire cohort, 4 were due to motion artefact: 2 in controlled HR group (in the right posterior descending artery and posterolateral ventricular branch in the same patient) $v s .2$ in elevated HR group: (in the mid left anterior descending artery and first diagonal branch in two different patients). The remaining segmental non-interpretability was due to severe calcium burden.

\section{Radiation dose}

There was no significant difference in median radiation 
Table 4 Analysis of image quality between groups

\begin{tabular}{|c|c|c|c|}
\hline Parameter & Group 1, (HR $\leq 60),(n=855)$ & Group 2, (HR 60-80), n=807 & $P$ value \\
\hline \multicolumn{4}{|l|}{ Likert score } \\
\hline Uninterpretable & $2(0.2 \%)$ & $6(0.7 \%)$ & 0.13 \\
\hline (I) Poor & $9(1.0 \%)$ & $13(1.6 \%)$ & 0.32 \\
\hline (III) Good & $258(30.2 \%)$ & $242(30.0 \%)$ & 0.93 \\
\hline (IV) Very good & $446(52.2 \%)$ & $380(47.1 \%)$ & 0.04 \\
\hline (V) Excellent & $119(13.9 \%)$ & $93(11.5 \%$ & 0.14 \\
\hline \multicolumn{4}{|l|}{ Predicted probabilities } \\
\hline (IV) Very good & 0.527 & 0.481 & \\
\hline (V) Excellent & 0.144 & 0.104 & \\
\hline Ordinal logistic regression $\beta$ coefficient & \multicolumn{2}{|c|}{$-0.371(95 \% \mathrm{Cl}:-0.57,-0.17)$} & $<0.01$ \\
\hline $\begin{array}{l}\text { Binary logistic regression } \beta \text { coefficient } \\
\text { (interpretable vs. non-interpretable) }\end{array}$ & \multicolumn{2}{|c|}{$-0.19(95 \% \mathrm{Cl}:-1.00,0.61)$} & 0.64 \\
\hline
\end{tabular}

Predicted probabilities as based on ordinal logit. Ordinal logistic regression co-efficient as based on ordinal outcome variable Likert 1 to 5. Binary logistic regression coefficient as based on non-interpretable (Likert <2) vs. interpretable (Likert $\geq 2$ ) segments.

dose between groups [2.45 (IQR 1.62-3.24) $\mathrm{mSv}$ in Group 1 vs. 2.70 (IQR 1.58-4.39) $\mathrm{mSv}$ in Group $2(\mathrm{P}=0.35)$ ] (Table 2). When stratified by HR of $\leq 60,61-70$ and $71-80 \mathrm{bpm}$, there was no difference in radiation dose (2.45 vs. 2.24 vs. $3.51 \mathrm{mSv}, \mathrm{P}=0.31)$. There remained no difference when the lowest cohort of HR $(\leq 60 \mathrm{bpm}) v s$. the highest (71-80 bpm) were compared, $\mathrm{P}=0.10$.

\section{Discussion}

We evaluated the diagnostic accuracy of second generation 320-CTCA in 107 consecutively enrolled patients with suspected CAD. Our results demonstrate that second generation 320-CTCA preserves diagnostic accuracy with HR up to $80 \mathrm{bpm}$ compared to traditional HR controlled patients $\leq 60 \mathrm{bpm}$. Diagnostic accuracy was preserved despite the high prevalence of obstructive $\mathrm{CAD}(67 \%)$ and diagnostic performance is similar to previously reported results in first generation 320-detector scanners (21). Our results do not advise liberal avoidance of heart rate control prior to CTCA, but rather serve as reassuring data in patients in whom rate control can be challenging or contraindicated. The results also demonstrate that image quality, and radiation dose may not be significantly compromised using the second generation scanner.

This is the first reported study of diagnostic accuracy in second generation 320-scanners. Previous studies have focused primarily on image quality and radiation exposure. Notably patients in this study underwent a scan protocol in which the acquisition window was widened when HR was elevated to allow for multiple phase reconstruction and evaluation. This obviated the need for 2 beat scans using multi-segment reconstruction which has described as a limitation in previous studies (22). Furthermore the AIDR $3 \mathrm{D}$ algorithm was incorporated into our protocol which may improve spatial resolution and reduce image noise to enhance image quality and lower radiation dose (23).

Surprisingly our findings indicate a comparable radiation dose in scans acquired during high and controlled heart rates. This may be secondary to the use of higher tube current and 
lower tube potential (24) to achieve sub-millisievert radiation dose scans (12). Furthermore, the faster gantry rotation speed of $275 \mathrm{~ms}$, allowing near isophasic imaging of the coronary tree avoids the need for multiple rotations. However, as mentioned our study utilized an increased phase window when HR >70 bpm to maximize phase capture. We did not find a difference in radiation dose across stratified HR cohorts, although there was clearly a numerical difference in those with $\mathrm{HR}>70 \mathrm{bpm}$ at 3.51 vs. $2.45 \mathrm{mSv}$ in those with HR $<60$ bpm, but this did not achieve statistical significance. Narrowing the phase window would further reduce radiation dosing although this requires confirmation with prospective testing.

Various Likert scoring systems have been used to report image quality in CTCA with recent studies comparing first and second generation quality differences, but not within scanner differences based on heart rate $(11,14)$. These scoring systems have generally been described in terms of means, however the use of mean values representing an ordinal scale that is likely to be non-normally distributed data is hard to interpret. We have reported medians to reflect the skewed distribution of Likert score, and additionally performed ordered logistic regression to better reflect statistical parameters of image quality. These methods demonstrate that increasing heart rate reduces the probability of achieving excellent image quality; however the difference in image scores between interpretable and non-interpretable segments is not significant.

The impact of reducing image quality with increasing heart rate, whilst not affecting overall stenosis severity, may have implications at a lesion level when analysing plaque characteristics. Therefore, pending further study, optimal heart rate control is necessary for plaque composition analysis.

In patients requiring HR control prior to 320-CTCA beta-blockers are most commonly used, however their use is contraindicated in chronic obstructive or reactive airways disease, and are avoided in those with hypotension (8). Up to $16 \%$ of patients presenting for CTCA have such contraindications $(6,7,9,10)$ whilst up to $50 \%$ will have an elevated HR $(7,10)$. Even with the addition of betablockade, up to $44 \%$ of patients may not be able to reach suggested target HR for protocol acceptable imaging (7). The demonstration of an ability of second-generation 320CTCA to accurately scan patients with HR up to $80 \mathrm{bpm}$ may not only result in less requirement for beta-blockade, but also increased clinical service delivery and efficiency.

\section{Limitations}

We acknowledge certain limitations to our study: this is a single-centre study of a selected and non-randomized patient population with vendor specific software and may not be generalizable to scanners with lower temporal resolution. Additionally, the study was reported by highly experienced cardiologists and therefore results may not be applicable to lower volume centres. The two groups are based on HR at presentation with subsequent beta-blockade if dictated by study protocol, providing two convenience samples rather than randomized groups. The relatively small numbers in each group may have resulted in a type II error with regards to no difference in radiation dose between groups. Furthermore, we excluded patients who had persistent atrial fibrillation. This is because our aim was to assess the influence of heart rate alone on diagnostic accuracy, without R-R interval variability. Finally, we did not assess plaque composition and only luminal stenosis which may impact lesion-level characterization.

\section{Conclusions}

In patients with suspected CAD, second-generation 320CTCA (Aquilion ONE) provides good overall diagnostic accuracy despite reduced image quality in patients with $\mathrm{HR}$ up to $80 \mathrm{bpm}$. There is a small, non-significant increase in radiation dose with patients at higher heart rates. Future study is required to assess plaque level characterization at elevated heart rates, as well as assess the potential improvement in departmental efficiency with a lower requirement for pre-scan beta-blockade.

\section{Acknowledgements}

None.

\section{Footnote}

Conflicts of Interest: The authors have no conflicts of interest to declare.

Ethical Statement: This study has been approved by the local human research ethics committee and therefore performed in accordance with the ethical standards laid down in the 1964 Declaration of Helsinki and its later amendments. All persons gave their informed consent prior to inclusion in the study. 


\section{References}

1. Dewey M, Zimmermann E, Deissenrieder F, et al. Noninvasive coronary angiography by 320 -row computed tomography with lower radiation exposure and maintained diagnostic accuracy: comparison of results with cardiac catheterization in a head-to-head pilot investigation. Circulation 2009;120:867-75.

2. de Graaf FR, Schuijf JD, van Velzen JE, et al. Diagnostic accuracy of 320-row multidetector computed tomography coronary angiography in the non-invasive evaluation of significant coronary artery disease. Eur Heart J 2010;31:1908-15.

3. Nasis A, Leung MC, Antonis PR, et al. Diagnostic accuracy of noninvasive coronary angiography with 320-detector row computed tomography. Am J Cardiol 2010;106:1429-35.

4. Kerl JM, Schoepf UJ, Zwerner PL, et al. Accuracy of coronary artery stenosis detection with CT versus conventional coronary angiography compared with composite findings from both tests as an enhanced reference standard. Eur Radiol 2011;21:1895-903.

5. Abbara S, Arbab-Zadeh A, Callister TQ, et al. SCCT guidelines for performance of coronary computed tomographic angiography: a report of the Society of Cardiovascular Computed Tomography Guidelines Committee. J Cardiovasc Comput Tomogr 2009;3:190-204.

6. de Graaf FR, Schuijf JD, van Velzen JE, et al. Evaluation of contraindications and efficacy of oral Beta blockade before computed tomographic coronary angiography. Am J Cardiol 2010;105:767-72.

7. Maffei E, Palumbo AA, Martini C, et al. "In-house" pharmacological management for computed tomography coronary angiography: heart rate reduction, timing and safety of different drugs used during patient preparation. Eur Radiol 2009;19:2931-40.

8. Mahabadi AA, Achenbach S, Burgstahler C, et al. Safety, efficacy, and indications of beta-adrenergic receptor blockade to reduce heart rate prior to coronary CT angiography. Radiology 2010;257:614-23.

9. Roberts WT, Wright AR, Timmis JB, et al. Safety and efficacy of a rate control protocol for cardiac CT. Br J Radiol 2009;82:267-71.

10. Shapiro MD, Pena AJ, Nichols JH, et al. Efficacy of prescan beta-blockade and impact of heart rate on image quality in patients undergoing coronary multidetector computed tomography angiography. Eur J Radiol 2008;66:37-41.
11. Tomizawa N, Maeda E, Akahane M, et al. Coronary CT angiography using the second-generation 320-detector row CT: assessment of image quality and radiation dose in various heart rates compared with the first-generation scanner. Int J Cardiovasc Imaging 2013;29:1613-8.

12. Chen MY, Shanbhag SM, Arai AE. Submillisievert median radiation dose for coronary angiography with a second-generation 320-detector row CT scanner in 107 consecutive patients. Radiology 2013;267:76-85.

13. Pelliccia F, Pasceri V, Evangelista A, et al. Diagnostic accuracy of 320-row computed tomography as compared with invasive coronary angiography in unselected, consecutive patients with suspected coronary artery disease. Int J Cardiovasc Imaging 2013;29:443-52.

14. Wong DT, Soh SY, Ko BS, et al. Superior CT coronary angiography image quality at lower radiation exposure with second generation 320-detector row CT in patients with elevated heart rate: a comparison with first generation 320-detector row CT. Cardiovasc Diagn Ther 2014;4:299-306.

15. Uehara M, Takaoka H, Kobayashi Y, et al. Diagnostic accuracy of 320-slice computed-tomography for detection of significant coronary artery stenosis in patients with various heart rates and heart rhythms compared with conventional coronary-angiography. Int J Cardiol 2013;167:809-15.

16. Austen WG, Edwards JE, Frye RL, et al. A reporting system on patients evaluated for coronary artery disease. Report of the Ad Hoc Committee for Grading of Coronary Artery Disease, Council on Cardiovascular Surgery, American Heart Association. Circulation 1975;51:5-40.

17. Van der Molen AJ, Joemai RM, Geleijns J. Performance of longitudinal and volumetric tube current modulation in a 64-slice CT with different choices of acquisition and reconstruction parameters. Phys Med 2012;28:319-26.

18. Hausleiter J, Meyer T, Hermann F, et al. Estimated radiation dose associated with cardiac CT angiography. JAMA 2009;301:500-7.

19. Leipsic J, Labounty TM, Heilbron B, et al. Adaptive statistical iterative reconstruction: assessment of image noise and image quality in coronary CT angiography. AJR Am J Roentgenol 2010;195:649-54.

20. Ropers U, Ropers D, Pflederer T, et al. Influence of heart rate on the diagnostic accuracy of dual-source computed tomography coronary angiography. J Am Coll Cardiol 2007;50:2393-8.

21. Li S, Ni Q, Wu H, et al. Diagnostic accuracy of 320-slice computed tomography angiography for detection of 
coronary artery stenosis: meta-analysis. Int J Cardiol 2013;168:2699-705.

22. Gang S, Min L, Li L, et al. Evaluation of CT coronary artery angiography with 320-row detector CT in a highrisk population. Br J Radiol 2012;85:562-70.

23. Irwan RN, S; Blum, A. AIDR 3D - Reduces Dose and Simultaneously Improves Image Quality. Toshina Med

Cite this article as: Nerlekar N, Ko BS, Nasis A, Cameron JD, Leung M, Brown AJ, Wong DT, Ngu PJ, Troupis JM. Seneviratne SK. Impact of heart rate on diagnostic accuracy of second generation 320-detector computed tomography coronary angiography. Cardiovasc Diagn Ther 2017;7(3):296-304. doi: $10.21037 / \mathrm{cdt} .2017 .03 .05$
Syst 2011:1-8.</bok>

24. Wong DT, Ko BS, Cameron JD, et al. Transluminal attenuation gradient in coronary computed tomography angiography is a novel noninvasive approach to the identification of functionally significant coronary artery stenosis: a comparison with fractional flow reserve. J Am Coll Cardiol 2013;61:1271-9. 